\title{
Breaking the Tie: How to Start Rehabilitation in Patients with Severe Limiting Intermittent Claudication
}

\section{Bassem Zarif ${ }^{1,2 *}$, Akram Samir ${ }^{1,2}$, Safwat Al-Nahrawi ${ }^{1,2}$ and Eman Gamal $^{2}$}

${ }^{1}$ National Heart Institute, Egypt

${ }^{2}$ Ministry of Health and Population, Egypt

*Corresponding Author: Bassem Zarif, Department of Cardiology, National Heart Institute, Ministry of Health and Population, Cairo, Egypt.
Received: October 18, 2021

Published: November 18, 2021

(C) All rights are reserved by Bassem Zarif., et al.

\begin{abstract}
Intermittent claudication (IC) is the most common symptom of peripheral artery disease (PAD). IC can worsen the quality of life and results in marked exercise intolerance and limitation to daily activities with increased risk of cardiovascular complications. Exercise training is the first line of conservative management in PAD. However, patients with IC may not tolerate weight-bearing exercises because of leg discomfort induced by physical effort. This review will address alternative rehabilitation strategies to reduce exercise limitations and improve exercise tolerance in patients with IC.
\end{abstract}

Keywords: Peripheral Artery Disease; Exercise Intolerance; Exercise Limitations; Intermittent Claudication; Exercise Training

\section{Introduction}

Around 200 million people around the world are living with peripheral artery disease (PAD) and the prevalence is rising strongly with elderly populations [9]. PAD is a systemic vascular affection rather than the coronaries by atherosclerotic changes [36], which occurs in more than one vascular bed, with increased atherothrombotic risk and the risk of microvascular disease [22]. As a final stage, it can present with chronic limb-threatening ischemia, which is characterized by ischemic rest pain and can progress to tissue necrosis [26]. Intermittent claudication (IC) is an exertional leg pain that causes the patient to stop walking until pain resolves [25]. There are two types of IC according to the underlying pathology, neurologic and vascular claudication, both produce activitylimiting symptoms in the legs. In neurogenic claudication, narrowing of lumbar spine results in nerve compression directly or indirectly through vascular compression of nerve roots [21]. While in vascular claudication, there is a narrowing of blood vessels results in a reduction of blood flow to extremities. This reduction in blood flow creates a mismatch between oxygen supply and metabolic demand (physical effort) causing ischemia in the legs, mainly the calf, thigh, or buttocks.

\section{Classification of peripheral arterial disease}

Many classifications were designed for PAD according to patient symptoms, angiographic findings, and incidence [12]:

\section{Fontaine classification}

This was the first classification for PAD depending on the patient symptoms only and classify the patient into four stages as follows, stage one is an organic disease without symptoms, stage 
two patient starts to develop symptoms and divided into two subgroups according to walking distance when patients develop leg pain, either more or less than 200 meters, stage three patient develop pain during rest, while stage four necrosis or gangrenous changes occurs in the limbs.

\section{Rutherford classification}

Regarding the incidence of ischemia, Rutherford classified PAD into either acute or chronic. Then include the clinical data to other investigation results as doppler, ankle brachial indices (ABI), and pulse volume recording for further classification of chronic limb ischemia. Acute limb ischemia classified as viable, threatened, or irreversibly damaged.

\section{Bollinger angiographic classification}

According to the site and severity of atherosclerotic lesions (anatomy of the arterial affection) identified by catheter angiography, each arterial segment was given a score of four for severity degree: total occlusion, luminal stenosis more than $50 \%$, stenosis 25 to $49 \%$ of the lumen, and plaques $<25 \%$ of the lumen. Also, several lesions were included: single lesion, multiple lesions less than half of the diseased segment, or multiple lesions more than half of the diseased segment. This classification used to decide the treatment method (Surgical or catheter angioplasty).

\section{Exercise limitations in PAD}

Reduced blood flow to lower limbs exaggerates intermittent claudication and functional disability. IC is the major limitation of exercise training in PAD. Additionally, PAD can lead to reduced muscle mass and mitochondrial dysfunction leading to muscle deconditioning and disuse (Figure 1). These symptoms reduce walking and functional capacity leading to physical inactivity and increase the risk of cardiovascular events [11]. Inflammation may accelerate functional impairment in PAD by favoring plaque growth and inducing skeletal muscle injury. Furthermore, endothelial dysfunction and reduced nitric oxide bioavailability may blunt blood flow during exercise [11].

\section{Management of peripheral arterial disease}

There is no specific medication to cure this condition, as this a longstanding arterial affection by multiple risk factors for atherosclerosis, including hypertension, smoking, obesity, and redundan-

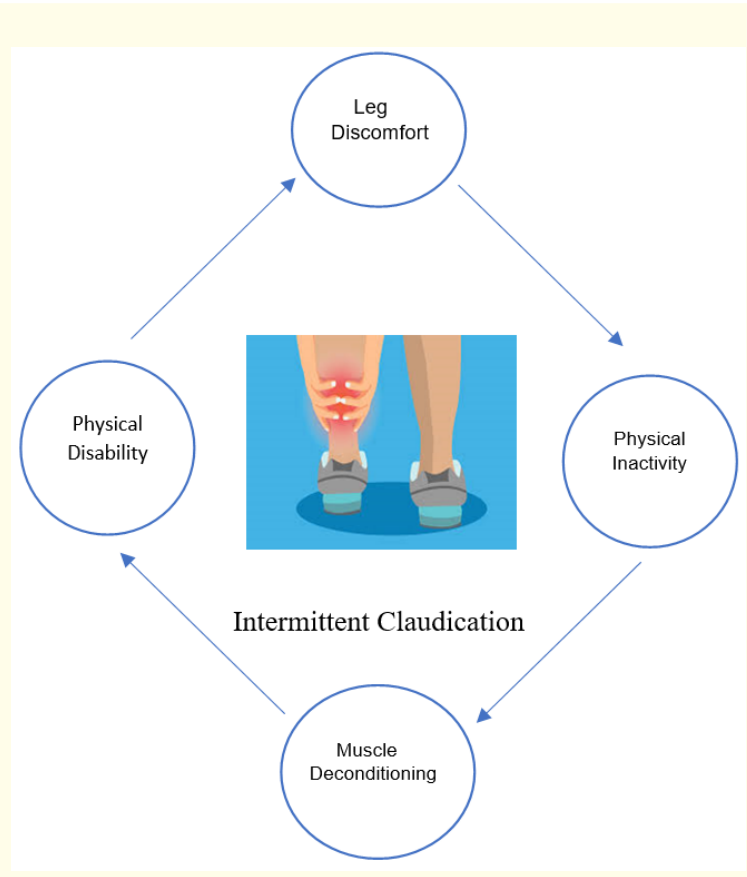

Figure 1: Redundant lifestyle result in muscular malfunctioning and physical disability leading to lower limb pain which may causes more physical redundancy as a vicious circle.

cy [32]. Management of chronic limb ischemia includes aggressive control of all risk factors, physical training, and revascularization. Medical therapy includes adjunctive medications using a platelet inhibitor or vitamin $\mathrm{K}$ antagonist (warfarin) may help after revascularization therapy [32]. Cilostazol is a new phosphodiesterase III inhibitor, a potent inhibitor of platelet aggregation with vasodilatory, antithrombotic, antiproliferative, and positive lipid-altering effects [29]. Pentoxifylline, another drug cause decreasing blood viscosity, improving red blood cells flexibility, and enhance microcirculatory flow and tissue oxygen concentration [28].

Exercise is a very well-known adjuvant therapy besides medical and revascularization treatment. Exercise added to standard care, resulted in improvement of symptomatology, different outcomes, and quality of life $[5,11,19,31]$.

\section{Exercise therapy with intermittent claudication}

Exercise training has the potential to reverse these pathologic events of IC and thereby interrupt the clinical course toward dis- 
ability [11]. In the middle 60s, unsupervised walking was demonstrated to improve claudication onset time and maximal walking distance (MWD) [16]. Recently, supervised walking has been reported as the gold standard training modality in the management of IC [18,35]. Patients are supervised and instructed to walk till claudication onset then rest until the pain is resolved [14,35]. Supervised treadmill walking has been shown to improve MWD and vascular function which in turn reduces the cardiovascular risk [14,18,35]. Comprehensive exercise interventions with PAD have several benefits including but not limited to functional capacity, claudication symptoms, and quality of life. The physiological mechanism behind exercise therapy in PAD is multifactorial, where exercise can reverse and suppress the inflammatory process of PAD and enhance physical capacity $[8,11]$. The following physiological adaptations can summarize the potential benefits of exercise training with IC:

\section{Vascular and metabolic adaptation}

The evidence that exercise training improves calf muscle blood flow in claudication is scarce and inconsistent with the improvement in walking capacity $[16,31]$. It is known that exercise improves ischemia and consequently IC by different mechanisms, potentially through recruitment of collaterals and stimulation of angiogenesis. Merits of exercise in improving functional limitations may exceed recruiting new collaterals by improving muscle metabolism, mitochondrial function, microcirculation, and endothelial function $[4,10]$. Thereby, enhanced muscle oxidative metabolism in response to exercise training can improve walking performance without change in muscle perfusion $[14,31]$. Furthermore, exercise training can contribute to the mitigation of claudication symptoms through reduced systemic inflammation and improve endothelial function [31]. However, the association between the anti-inflammatory effect of exercise training with the improvement in walking capacity is unclear yet [31]. Further studies are required to assess the direct effect of exercise training on walking capacity and claudication symptoms mediated through vascular adaptations.

\section{Cardiorespiratory adaptations}

Cardiorespiratory fitness can defer the onset of claudication pain during walking in PAD and contributes to improved walking performance without changes in ABI $[5,34,38]$. Hence, improved general physical conditioning and aerobic capacity may contribute to the improved walking ability alongside metabolic adaptations [5].

\section{Neural adaptation}

Peripheral neuropathy can be associated with PAD in form of axonal and myelin damage that may cause muscle spasticity, muscle atrophy, and strength loss with further disability and claudication worsening [7]. Exercise training can enhance the sensory and motor function of peripheral nerves, and reduce associated neuropathic pain and chronic neuroinflammation. Consequently, these adaptations may alter the pain threshold in favor of improving claudication distance and exercise capacity $[6,7]$.

\section{Exercise prescription in PAD with severely limiting IC}

In this section, we will focus on the dilemma between exertional leg symptoms induced by exercise, and how to tackle this cycle (Figure 1) to reduce physical inactivity and improve exercise tolerance in patients with severe claudication symptoms. In fact, some patients with advanced IC cannot tolerate walking because of persistent leg pain and severe muscular ischemia [35]. Also, claudication symptom is a major barrier that impedes adherence to the walking training program [1]. In line with this, it has been reported that exercise training can reduce walking performance and mitochondrial capacity in some patients with advanced PAD [37]. Additionally, patients with several comorbidities can have fewer improvements in walking distance after supervised exercise training [8]. Consequently, the role of exercise and healthcare professionals is to tailor alternative training strategies alongside walking to reduce the physical impact on lower arteries and improve exercise tolerance simultaneously. Several exercise interventions can be beneficial for all patients with IC despite the severity of the condition [35]. Examples of these alternative modalities that can be prescribed for patients with severe IC are summarized in table 1 including:

\section{Resistance training}

Patients with IC can have less muscle mass and lower strength $[13,19]$. Resistance training (RT) can improve walking capacity and endurance $[20,27]$. RT increases muscle fiber area and capillarization to muscle, which in turn improves oxygen delivery and promotes walking endurance in symptomatic patients with PAD after long-term (24 weeks) resistance training [20]. In the middle of the 90s, Hiatt and colleagues reported the superiority of treadmill walking to resistance training in terms of walking performance and tolerance [15]. In contrast, it has been shown that wholebody RT can improve walking capacity and claudication symptoms similar to walking training $[20,24,27]$. Few studies investigate the 
combined RT with aerobic training and found no additional improvement in MWD compared to isolated aerobic training [15,17]. However, some patients reported less pain during RT compared to walking training [27]. Reduced pain during RT can be beneficial for claudicants with physical and social walking barriers, which can increase their engagement to regular physical activity with similar training adaptations to walking.

\section{Cycling}

Leg cycling has been shown to elicit similar cardiovascular and metabolic strain to treadmill walking in patients with IC [3]. However, the prescription of cycling as an alternative mode to walking has little evidence yet, and there are controversial findings in the literature. In the previous decay, Sanderson and colleagues reported that treadmill walking is more effective than cycling for the improvement of walking endurance and tolerance [30]. On the contrary side, Walker and colleagues have shown that arm and leg cycling can improve walking distance and mitigate claudication symptoms similarly [38]. Different factors can contribute to this controversy. For instance, the modalities of exercise testing used for MWD were different among the aforementioned trials (graded treadmill walking vs incremental shuttle test). Further clinical trials are required to validate the accuracy and reliability of exercises test used with PAD patients. Surprisingly, arm cycling can improve walking distance in patients with IC, this finding was attributed to systemic adaptations of cardiorespiratory fitness [5,34,38]. Furthermore, improved walking capacity was associated with peak cardiorespiratory fitness [5,34]. Arm cycling can be prescribed to patients with severe PAD and comorbidities to improve exercise tolerance with potential progression to leg cycling and walking.

Table 1: Exercise prescription for patients with IC.

\begin{tabular}{|c|c|c|c|c|c|}
\hline Type & Intensity & Duration & $\begin{array}{c}\text { Work-to-rest } \\
\text { Ratio }\end{array}$ & Frequency & Progression \\
\hline $\begin{array}{l}\text { Interval walking } \\
\text { (exercise-rest- } \\
\text { exercise) } \\
{[2,25,35]}\end{array}$ & $\begin{array}{ll}\text { - } & 40-60 \% \text { of max workload on } \\
\text { Or } & \text { treadmill test } \\
\text { - } & \begin{array}{l}\text { The workload that elicits } \\
\text { claudication within } 3-5 \text { min } \\
\text { on 6MWT }\end{array} \\
\text { Or } \quad \text { Moderate to moderately } \\
\text { - } \\
\text { severe claudication on the } \\
\text { claudication scale }\end{array}$ & $30-60 \mathrm{~min}$ & 5-10: $2-5 \mathrm{~min}$ & 3-5 d/week & $\begin{array}{l}\text { Every } 1-2 \text { weeks duration } \\
\text { increases ( } 5 \mathrm{~min} / \text { day) to } \\
\text { reach } 60 \mathrm{~min}\end{array}$ \\
\hline $\begin{array}{l}\text { Resistance Training } \\
{[2,24,25]}\end{array}$ & $20-50 \% 1 \mathrm{RM}$ & $30-60 \mathrm{~min}$ & NA & $2 \mathrm{~d} /$ week & $\begin{array}{c}\text { Over } 4 \text { sessions to reach } \\
30-80 \% 1 \mathrm{RM}\end{array}$ \\
\hline
\end{tabular}

6MWT: six-minute-walk test, $\mathrm{VO}_{2}$ peak: peak oxygen uptake, $1 \mathrm{RM}$ : one repetition maximum, Max: maximal, Min: minutes, d: days , NA: not available. 


\section{Conclusion}

Exercise therapy consists of multiple interventions that need to be personalized and tailored to meet the patient's condition and tolerance. In context with IC in PAD, walking is a gold standard training of IC management, and feasible in most patients. Several research studies looked for other alternative exercise modes to be implemented for those who cannot tolerate walking, and for those who cannot attain sufficient physiological adaptations because of severe limiting IC. One of the most promising lines of those rehabilitation modalities is the implementation of arm, leg cycling, and whole-body RT alongside walking in patients with markedly limiting IC, however, further research is needed to ensure the effectiveness of this rehabilitation model.

\section{Conflict of Interest}

None declared.

\section{Bibliography}

1. Abaraogu U., et al. "Barriers and enablers to walking in individuals with intermittent claudication: A systematic review to conceptualize a relevant and patient-centered program". PLoS One 13 (2018): e0201095.

2. Ambrosetti M., et al. "Secondary prevention through comprehensive cardiovascular rehabilitation: From knowledge to implementation. 2020 update. A position paper from the Secondary Prevention and Rehabilitation Section of the European Association of Preventive Cardiology". European Journal of Preventive Cardiology (2020): 2047487320913379.

3. Askew C D., et al. "Physiological and symptomatic responses to cycling and walking in intermittent claudication". Clinical Physiology and Functional Imaging 22 (2002): 348-355.

4. Blomqvist C G and B Saltin. "Cardiovascular adaptations to physical training". Annual Review of Physiology 45 (1983): 169-189.

5. Bronas U G., et al. "Comparison of the effect of upper body-ergometry aerobic training vs treadmill training on central cardiorespiratory improvement and walking distance in patients with claudication". Journal of Vascular Surgery 53 (2011): 1557-1564.

6. Cheng J., et al. "Treadmill exercise promotes neurogenesis and myelin repair via upregulating Wnt/betacatenin signaling pathways in the juvenile brain following focal cerebral ischemia/reperfusion". International Journal of Molecular Medicine 45 (2020): 1447-1163.

7. Dobson JL., et al. "Benefits of exercise intervention in reducing neuropathic pain". Frontiers in Cell Neuroscience 8 (2014): 102.

8. Dörenkamp Sarah., et al. "Patient Characteristics and Comorbidities Influence Walking Distances in Symptomatic Peripheral Arterial Disease: A Large One-Year Physiotherapy Cohort Study". PLoS One 11 (2016): e0146828-e28.

9. Fowkes F G., et al. "Comparison of global estimates of prevalence and risk factors for peripheral artery disease in 2000 and 2010: a systematic review and analysis". Lancet 382 (20213): 1329-1340.

10. Haas T L., et al. "Exercise training and peripheral arterial disease". Comprehensive Physiology 2 (2012): 2933-3017.

11. Hamburg N M and GJ Balady. "Exercise rehabilitation in peripheral artery disease: functional impact and mechanisms of benefits". Circulation 123 (2011): 87-97.

12. Hardman R L., et al. "Overview of classification systems in peripheral artery disease". Seminars in Interventional Radiology 31 (2014): 378-388.

13. Harwood A E., et al. "A systematic review of muscle morphology and function in intermittent claudication". Journal of Vascular Surgery 66 (2017): 1241-1257.

14. Hiatt W R., et al. "Benefit of exercise conditioning for patients with peripheral arterial disease". Circulation 81 (1999): 602609.

15. Hiatt W R., et al. "Superiority of treadmill walking exercise versus strength training for patients with peripheral arterial disease. Implications for the mechanism of the training response". Circulation 90 (1994): 1866-1874.

16. Larsen $\mathrm{O} A$ and $\mathrm{N} A$ Lassen. "Effect of daily muscular exercise in patients with intermittent claudication". Lancet 2 (1996): 1093-1096.

17. Machado I., et al. "Combined Aerobic and Resistance Exercise in Walking Performance of Patients With Intermittent Claudication: Systematic Review". Frontiers in Physiology 10 (2019): 1538. 
18. McDermott M M., et al. "Treadmill exercise and resistance training in patients with peripheral arterial disease with and without intermittent claudication: a randomized controlled trial". JAMA 301 (2009): 165-174.

19. McDermott Mary McGrae., et al. "Leg strength in peripheral arterial disease: associations with disease severity and lower-extremity performance". Journal of Vascular Surgery 39 (2004): 523-530.

20. McGuigan M R., et al. "Resistance training in patients with peripheral arterial disease: effects on myosin isoforms, fiber type distribution, and capillary supply to skeletal muscle". Journals of Gerontology Series A Biological Sciences and Medical Science 56 (2001): B302-310.

21. Nadeau M., et al. "The reliability of differentiating neurogenic claudication from vascular claudication based on symptomatic presentation". Canadian journal of surgery. Journal Canadien De Chirurgie 56 (2013): 372-377.

22. Olesen K K W., et al. "Peripheral artery disease, lower limb revascularization, and amputation in diabetes patients with and without coronary artery disease: a cohort study from the Western Denmark Heart Registry". BMJ Open Diabetes Research Care 9 (2021): e001803.

23. Parmenter B J., et al. "Resistance training as a treatment for older persons with peripheral artery disease: a systematic review and meta-analysis". British Journal of Sports Medicine 54 (2020): 452-461.

24. Parmenter BJ., et al. "High-intensity progressive resistance training improves flat-ground walking in older adults with symptomatic peripheral arterial disease". Journal of the American Geriatrics Society 61 (2013): 1964-1970.

25. Pescatello Linda S. ACSM's guidelines for exercise testing and prescription (Wolters Kluwer/Lippincott Williams and Wilkins Health: Philadelphia) (2014).

26. Peters C M L., et al. "Two-year Outcome of Quality of Life and Health Status for the Elderly with Chronic Limb-threatening Ischemia". Clinical Interventions in Aging 15 (2020): 23832395.

27. Ritti-Dias R M., et al. "Strength training increases walking tolerance in intermittent claudication patients: randomized trial". Journal of Vascular Surgery 51 (2010): 89-95.
28. Salhiyyah K., et al. "Pentoxifylline for intermittent claudication". Cochrane Database of Systematic Reviews 9 (2015): CD005262.

29. Samra S S., et al. "Efficacy and safety of cilostazol, a novel phosphodiesterase inhibitor in patients with intermittent claudication". Journal of Indian Medical Association 101 (2003): 561562,64 .

30. Sanderson B., et al. "Short-term effects of cycle and treadmill training on exercise tolerance in peripheral arterial disease". Journal of Vascular Surgery 44 (2006): 119-127.

31. Stewart K J., et al. "Exercise training for claudication". The New England Journal of Medicine 347 (2002): 1941-1951.

32. Swedish Council on Health Technology, Assessment. SBU Systematic Review Summaries.' in, Peripheral Arterial Disease - Diagnosis and Treatment: A Systematic Review (Swedish Council on Health Technology Assessment (SBU) (2008).

33. Copyright (C) 2007 by the Swedish Council on Health Technology Assessment.: Stockholm) (2007).

34. Treat-Jacobson D., et al. "Efficacy of arm-ergometry versus treadmill exercise training to improve walking distance in patients with claudication". Vascualr Medicine 14 (2009): 203213.

35. Treat-Jacobson D., et al. "Optimal Exercise Programs for Patients With Peripheral Artery Disease: A Scientific Statement From the American Heart Association". Circulation 139 (2019): e10-e33.

36. Ugwu E., et al. "Ankle brachial index as a surrogate to vascular imaging in evaluation of peripheral artery disease in patients with type 2 diabetes". BMC Cardiovasc Disord 21 (2021): 10.

37. van Schaardenburgh M., et al. "Exercise in claudicants increase or decrease walking ability and the response relates to mitochondrial function". Journal of Translational Medicine 15 (2017): 130.

38. Walker R D., et al. "Influence of upper- and lower-limb exercise training on cardiovascular function and walking distances in patients with intermittent claudication". Journal of Vascular Surgery 31 (2000): 662-669.

\section{Volume 5 Issue 12 December 2021 (C) All rights are reserved by Bassem Zarif., et al.}

